\title{
Mesoscale temperature fluctuations in the stratosphere
}

\author{
B. L. Gary \\ Jet Propulsion Laboratory, Pasadena, CA 91109, 5320 E. Calle Manzana, Hereford, AZ 85615, USA
}

Received: 18 May 2006 - Published in Atmos. Chem. Phys. Discuss.: 4 August 2006

Revised: 4 October 2006 - Accepted: 9 October 2006 - Published: 12 October 2006

\begin{abstract}
An airborne instrument that measures altitude temperature profiles is ideally suited for the task of characterizing statistical properties of the vertical displacement of isentrope surfaces. Prior measurements of temperature fluctuations during level flight could not be used to infer isentrope altitude variations because lapse rate information was missing. The Microwave Temperature Profiler instrument, which includes lapse rate measurements at flight level as a part of temperature profiles, has been used on hundreds of flights to produce altitude versus ground track crosssections of potential temperature. These cross-sections show isentrope altitude variations with a horizontal resolution of $\sim 3 \mathrm{~km}$ for a $>6 \mathrm{~km}$ altitude region. An airborne isentropealtitude cross-section (IAC) can be compared with a counterpart IAC generated from synoptic scale data, based on radiosondes and satellite instruments, in order to assess differences between the altitudes of isentrope surfaces sampled at mesoscale versus synoptic scale. It has been found that the synoptic scale isentropes fail to capture a significant component of vertical displacement of isentrope surfaces, especially in the vicinity of jet streams. Under the assumptions that air parcels flow along isentrope surfaces, and change temperature adiabatically while undergoing altitude displacements, it is possible to compute mesoscale temperature fluctuations that are not present in synoptic scale back trajectory parcel temperature histories. It has been found that the magnitude of the mesoscale component of temperature fluctuations varies with altitude, season, latitude and underlying topography. A model for these dependences is presented, which shows, for example, that mesoscale temperature fluctuations increase with altitude in a systematic way, are greatest over mountainous terrain, and are greater at polar latitudes during winter.
\end{abstract}

\footnotetext{
Correspondence to: B. L. Gary

(blgary@umich.edu)
}

\section{Introduction}

The Microwave Temperature Profiler (MTP) was developed by NASA at the Jet Propulsion Laboratory (JPL) during the 1970s for airborne studies of clear air turbulence. The MTP is the only airborne sensor system that provides altitude temperature profiles throughout an altitude region that extends from several kilometers below to several kilometers above flight level, and these measurements are provided in realtime at intervals of 2 to $5 \mathrm{~km}$ along the flight path. To date various MTP instruments have flown on 7 aircraft (CV990, C141, ER-2, DC-8, WB57, L118C, M55) for 46 atmospheric research missions, 739 flights and have accumulated 4176 flight hours of data.

In response to the discovery of the "ozone hole" in 1985 (Farman et al.) NASA organized an airborne mission to study the ozone depletion process (Airborne Antarctic Ozone Experiment, or AAOE). One of the NASA atmospheric research aircraft used for AAOE was the high-flying NASA ER-2 (19 to $21 \mathrm{~km})$. An MTP was included in the ER-2 payload for this first ozone hole mission, and it has been included in all subsequent NASA missions involving the ER-2 for the study of ozone depletion. An MTP for the NASA DC-8 atmospheric research aircraft has been included in the payload for all airborne ozone depletion missions since 1991. The present analysis is based on data from a subset of the ER-2 and DC-8 ozone depletion flights from 1988 to 2001.

As with any instrument that measures something for the first time new and unexpected things have been discovered from MTP data. The MTP aboard a NASA ER-2 aircraft was used in 1987 to show that mountain waves penetrate the polar vortex tropopause and amplify with altitude in the lower stratosphere in approximate agreement with a theoretical model (Gary, 1989). In 1987 and 1989 the same MTP measured an ever-present background of vertical displacements of isentrope surfaces having mesoscale spatial frequencies. At polar latitudes, during winter, the amplitude of this structure was found to be greater for flight over land than over ocean (Gary, 1989; Bacmeister and Gary, 1990). Some

Published by Copernicus GmbH on behalf of the European Geosciences Union. 
of these findings have been reported in the literature but this is the first report of a systematic study of MTP-measured mesoscale fluctuations versus latitude, season, altitude and underlying terrain.

When altitude fluctuations of isentropes (surfaces of constant potential temperature) were first reported at an ozone hole mission workshop in 1991 there was widespread skepticism about their existence. In retrospect the mesoscale component should not have been a surprise since earlier studies of temperature recordings using commercial airplanes (Nastrom and Gage, 1985; Gage and Nastrom, 1986) showed that isentrope surfaces were not smooth. However, these level-flight studies could not assign altitude displacements to the isentropes because lapse rate information was lacking. The MTP provides this missing information, and allows all nearby isentrope surfaces to be assigned reliable altitudes along the flight path.

The concern at the 1991 workshop over the mesoscale temperature fluctuations required by MTP measurements was motivated by the fact that models were being used to study the formation and evolution of polar stratospheric clouds (PSCs) using back trajectories derived from an assimilated data base for temperature and wind fields. Since assimilated data is based on radiosonde and satellite measurements they are limited to spatial wavelengths longer than about $400 \mathrm{~km}$. Hence, the back trajectories used to study the role of PSCs in ozone depletion did not include mesoscale temperature fluctuations, and it was not known whether this was a serious limitation for the model studies.

The airborne MTP can be used to derive the temperature field within an altitude/ground track cross-section, and this provides an ideal means for evaluating the magnitude of the missing short time scale temperature fluctuations. Several studies of the implications of mesoscale temperature fluctuations and the microphysics of polar stratospheric cloud formation and evolution have been published (Wofsy et al., 1993; Murphy and Gary, 1995; Tabazedeh et al., 1996; Carslaw et al., 1998; Voigt et al., 2000; Gao et al., 2001; Doernbrack et al., 2002; Fueglistaler et al., 2003; Murphy, 2003; Karcher and Strom, 2003; Hoyle et al., 2005).

Murphy and Gary (1995) use microphysical arguments involving time constants for various effects to conclude that rapid temperature fluctuations should affect the nucleation of polar stratospheric cloud droplets, and the large cooling rates experienced by air parcels have important implications for denitrification and dehydration. Murphy (2003) has shown the importance of superimposing realistic mesoscale temperature fluctuations upon synoptic temperature variations in a model for ice cloud evolution that involves the conversion of metastable cubic ice crystals to normal hexagonal ice crystals. This leads to a smaller number of ice crystals which then grow large enough for their faster fall velocities to dehydrate the cloud layer. Karcher and Strom (2003) deduce that the high values for measured ice crystal number density in young cirrus clouds can be explained by their measure- ments of a mesoscale component of vertical winds that produce temperature fluctuations much greater than inferred by synoptic scale simulations. Hoyle et al. (2005) quantify the characteristics of the fluctuations in terms of energy spectral density and amplitudes. The magnitude of these mesoscale temperature fluctuations is in agreement with the findings of this study.

One purpose of this publication is to make available information on the magnitude of short time scale temperature variations so that model studies that employ back trajectories can include realistic mesoscale effects.

A second shortcoming of the synoptic temperature field is that it can have large errors in the vicinity of jet streams. Satellite measurements of the temperature field at spatial scales slightly shorter than synoptic have been reported by $\mathrm{Wu}$ and Waters (1996a, b). These measurements imply that vertical motions with horizontal wavelengths at the long end of the mesoscale spectrum have greater amplitudes in the vicinity of jets and over high mountain ranges. The MTP supports these findings using even shorter spatial frequency information.

A third use for the quantitative model for mesoscale altitude structures presented here is the shortcoming of current model studies of global circulation to accurately estimate the amount of momentum that is transferred to the mesosphere and upper stratosphere by breaking waves. Bacmeister (1993) pointed out the importance of being able to predict the existence of atmospheric mountain waves and their amplitude growth with altitude in order to calculate the altitude and magnitude of the momentum flux. The MTP-based model for predicting most likely vertical wave amplitude from a known setting (described below) allows for an additional input to models that need to incorporate likely wave breakdown from the ever-present component of mesoscale vertical motions.

MTP measurements have been made for a wide range of altitudes ( 7 to $22 \mathrm{~km}$ ), latitudes ( -72 to +80 degrees), seasons (all months) and underlying topography. The present analysis is restricted to the Northern Hemisphere. Isentrope altitudes having mesoscale resolution are compared with isentrope altitudes having only synoptic scale resolution in order to assess the amplitude of the mesoscale component. The mesoscale amplitude is then correlated with several possible independent variables to determine which ones can be used to predict mesoscale amplitude. It is found that most of the observed differences of mesoscale amplitude from one flight segment to another can be accounted for using four independent variables: altitude, latitude, season and underlying topography.

The scope of this article is limited to a description of the magnitude of mesoscale temperature (and altitude) fluctuations. No attempt is made to translate this information to implications of the three possible uses for it noted in this introduction section (back trajectory temperature calculations, missing mesoscale structures near jet streams, and 
momentum transfer to the mesosphere and upper stratosphere caused by Kelvin-Helmholtz wave breakdown). An assessment of these and other possible implications of the ever-present mesoscale vertical motions is a task for qualified theoretical atmospheric scientists and should not be attempted by an "observationalist" such as this author.

\section{Remote sensing concept}

The MTP consists of a microwave radiometer that measures the intensity of thermal radiation from the atmosphere at frequencies where oxygen molecules are highly absorptive and emissive. The frequency region 52 to $59 \mathrm{GHz}$ is ideal for this purpose, since oxygen absorption is dominant and varies two orders of magnitude within this frequency range. At these frequencies water vapor and liquid water droplets have negligible effects in the upper troposphere and lower stratosphere. At typical altitudes there is sufficient oxygen to produce an optical depth of greater than one for views at all elevation angles. Therefore, the microwave "brightness temperature" corresponds, to first order, to the physical temperature of the air at a distance (the "applicable range") where the weighting function of thermal emission is approximately 1/e.

The various MTP instruments used to date operate at either 2 or 3 frequencies. For example, the MTP aboard the NASA DC-8, referred to hereafter as the MTP/DC8 instrument, operates at 55.51, 56.66 and $58.79 \mathrm{GHz}$. At typical altitudes these frequencies afford applicable ranges of 2.8, 1.1 and $0.6 \mathrm{~km}$. A horn antenna with a half-power beam width of $\sim 7.5$ degrees is scanned through a set of 10 elevation angles, ranging from near nadir to near zenith. In this way the MTP samples air throughout an altitude region extending from more than $2.8 \mathrm{~km}$ below the aircraft to more than $2.8 \mathrm{~km}$ above the aircraft. Since the measurements are influenced by thermal emission at distances beyond the applicable range it is possible to recover air temperature information throughout an altitude region with a thickness much greater than twice the applicable range. A brief description of the hardware can be found in Denning et al. (1989).

Many procedures can be used to convert brightness temperature "observables" to "retrievables" that define a profile of air temperature versus altitude, $\mathrm{T}(\mathrm{z})$, as described in Gary (1989). The simplest procedure is to treat each observed brightness temperature as an air temperature at an altitude given by "applicable range times sine of elevation angle". This was done during the first years of using the MTP. Much better performance can be achieved by using statistical retrieval procedures. This approach requires that a large set of radiosondes be used to calculate what an MTP would observe if it were perfectly calibrated; a multiple regression analysis is then performed to derive a set of coefficients that allow the temperature at an altitude of interest to be derived by multiplying an observable vector by an appropriate set of coefficients. A set of coefficients is obtained for each altitude of interest, which is called a "retrieval coefficient matrix". Each row in this matrix corresponds to an altitude, so with many rows of retrieval coefficients there will be many altitudes of derived air temperature. For the data of this analysis it was possible to derive $\mathrm{T}(\mathrm{z})$ profiles using the MTP/DC8 that exhibit an accuracy of $<1.2 \mathrm{~K}$ from 8 to $13 \mathrm{~km}$ (pressure altitude), and $<2.0 \mathrm{~K}$ from 6 to $17.5 \mathrm{~km}$. These accuracy estimates are based on comparisons of MTP T(z) profiles with radiosonde profiles taken when the aircraft passed close to a radiosonde launch site (allowing for temporal interpolation between preceding and following radiosondes). Improved performance for a greater range of altitudes has been achieved for recent flight data by Mahoney and Gary (2003). For the purposes of this investigation these improvements are not important since only isentropes at nearby altitudes are used to establish mesoscale fluctuation statistics.

\section{Tropical example of mesoscale versus synoptic scale isentropes}

Figure 1 is used to show differences between synoptic scale and mesoscale "isentrope altitude cross-sections" (IACs). The traces show the altitude of potential temperature surfaces versus latitude along a ground track flown by NASA's ER-2 aircraft on the date 27 March 1994. The smooth thick traces are a synoptic scale IAC derived from a file of temperature versus altitude along the flight path that was produced by Schoeberl et al. (1994) from a 3-dimensional field of assimilated data (based on radiosonde and satellite temperature measurements). The file produced by Schoeberl et al. (1994) of the NASA Goddard Space Flight Center were in support of the NASA-sponsored mission called "Airborne Southern Hemisphere Ozone Experiment/Measurement for Assessing the Effects of Stratospheric Aircraft" (ASHOE/MAESA).

The mesoscale IAC in Fig. 1 is shown by thin traces with altitude structure that should not be mistaken for "noise". They were constructed from measurements by an MTP instrument aboard the NASA ER-2 aircraft, hereafter referred to as the MTP/ER2 instrument. Since the tropopause was at $16 \mathrm{~km}$ for this IAC the entire IAC in this figure is for the stratosphere. The flight track "curtain cross-section" is oriented approximately north-south for a flight from Hawaii (right side) to Fiji (left side). Although the wind direction for these data is "out of the page", it can nevertheless be used to estimate the magnitude of isentrope slope values in an orthogonal (parallel to the wind) cross-section. This can be done since cross-sections at a given location typically exhibit isentropes with a similar "wrinkle character" in crosssections of all azimuths, provided mountain waves are not present.

Considering first the synoptic scale isentropes in Fig. 1, and assuming adiabatic behavior for air parcels as they change altitude, this figure can be used to deduce that as air parcels moved through this region they underwent altitude 


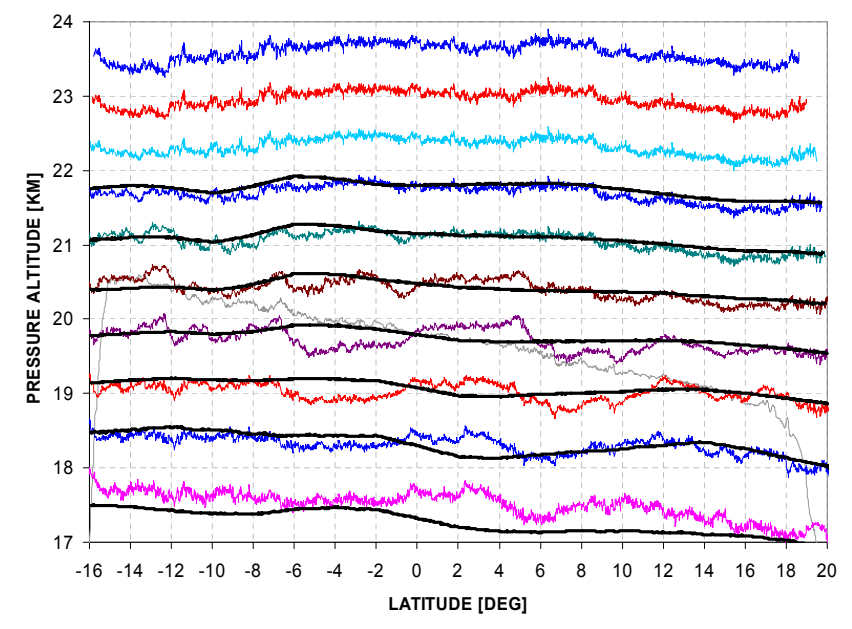

Fig. 1. Isentrope altitude cross-sections for an ER-2 flight from Hawaii to Fiji, 27 March 1994. The isentropes are $20 \mathrm{~K}$ of potential temperature apart starting with $400 \mathrm{~K}$ at $\sim 17.5 \mathrm{~km}$. The ER-2 altitude is shown by the light-gray trace. The thick, smooth black traces are isentropes based on synoptic scale (assimilated) data. The thin colored traces, with detailed structure, are isentropes based on MTP/ER2 data.

excursions of $\sim 100 \mathrm{~m}$ with periods corresponding to spatial wavelengths of about $900 \mathrm{~km}$ ( 8 arc degrees). The Meteorology Measurement System, MMS, (Scott et al., 1990) measured the wind speed in this region to be $10 \mathrm{~m} / \mathrm{s}$, eastward. Combining this horizontal wind speed with the synoptic isentrope structure leads to air parcel altitude variations of $\sim 100 \mathrm{~m}$ (temperature fluctuations $\sim 1 \mathrm{~K}$ ) with periods of $\sim 25 \mathrm{~h}(900 \mathrm{~km}$ divided by $10 \mathrm{~m} / \mathrm{s})$. Adiabatic heating and cooling rates for such air parcels would be $\sim 2$ or $3 \mathrm{~K} /$ day. Trajectory analyses based on assimilated data for this region therefore imply that air parcels would undergo small temperature changes, with time scales of 1-day and longer, and experience relatively benign heating and cooling rates.

Considering the mesoscale isentropes in Fig. 1, air parcels following isentropes would rise and fall by much greater amounts and with shorter timescales. Heating and cooling rates of several hundred K/day, maintained for tens of minutes, are inferred to exist at many locations. The airborne MTP/ER2 instrument measures an altitude profile of temperature every $10 \mathrm{~s}$ (for $1994 \mathrm{data}$ ). This corresponds to $2.1 \mathrm{~km}$ (or 0.02 degrees of latitude for north/south flight). Each $\mathrm{T}(\mathrm{z})$ profile extends from approximately $4 \mathrm{~km}$ below flight altitude to $4 \mathrm{~km}$ above. Near flight level, where the MTP is most accurate, the RMS difference between the mesoscale and synoptic scale altitudes is $170 \mathrm{~m}$, and the maximum difference is $390 \mathrm{~m}$. It is also apparent that the highest spatial frequencies in the synoptic plots are misleading, which is to say that about half the time the small structures in the synoptic isentropes do NOT correlate with features in the measured isentropes. Because of the dramatic differences between the heating and cooling rates for air parcels traveling along mesoscale and synoptic scale isentropes, and because of the occasionally large altitude discrepancies of the two isentrope types, it is appropriate to ask whether the MTPmeasured mesoscale isentropes are accurate.

\section{Accuracy of MTP-based isentrope altitudes}

There is always excellent agreement between the MTP temperature profile's temperature at flight level and the MMS in situ air temperature record when the MMS $1 \mathrm{~Hz}$ data is smoothed to correspond to the slower sampling of the MTP. The two temperature series agree, with biases typically $\sim 0.3 \mathrm{~K}$ and smaller differences on short time scales (i.e., small offsets may exist, but the temporal structures are essentially identical). This agreement assures that the MTP isentrope altitude structures near flight level are accurate (i.e., that their vertical variations are correct). An additional consistency check can be performed by combining MMS in situ temperature with MTP lapse rate to calculate isentrope altitudes near flight level. When this is done the two sets of IACs near flight level are invariably in excellent agreement, with an RMS difference of $\sim 35 \mathrm{~m}$. This implies that the MTP isentrope altitude accuracy is better than $35 \mathrm{~m}$ near flight altitude.

Comparisons have been made of MTP measurements with radiosonde-based predictions of what MTP should measure for times when the aircraft flies close to radiosonde sites. Based on these comparisons it is estimated that RMS accuracy of MTP temperature profiles is $<1.0 \mathrm{~K}$ for a $3 \mathrm{~km}$ altitude region centered upon flight altitude, and that at the extremities of an $8 \mathrm{~km}$ region (centered upon flight altitude) the RMS accuracy of MTP T(z) profiles is $\sim 2.5 \mathrm{~K}$. For flight in an isothermal stratosphere, for example, MTP temperature profiles can therefore be used to determine isentrope altitudes with an accuracy of $<100 \mathrm{~m}$ near flight altitude, $<250 \mathrm{~m}$ at $4 \mathrm{~km}$ above and below flight altitude (with intermediate accuracy for intermediate altitudes). For typical polar ozone conditions, with $\mathrm{dT} / \mathrm{dZ}=-2 \mathrm{~K} / \mathrm{km}$, the isentrope altitude accuracies are $130 \mathrm{~m}$ and $320 \mathrm{~m}$.

Since precision is always better than accuracy, assuming calibration errors vary slower than measurement intervals, the altitude structure of isentropes is better than would be implied by the accuracies just quoted. A conservative estimate of MTP isentrope altitude precision is $\sim 30 \mathrm{~m}$ near flight altitude (based on comparisons with MMS) and $\sim 100 \mathrm{~m}$ at $4 \mathrm{~km}$ above and below flight altitude. These precision estimates are compatible with known MTP stochastic measurement uncertainties. The implication of the analyses of MTP accuracy and precision is that essentially all of the MTP-derived mesoscale structure in Fig. 1 is statistically significant and is therefore real. 


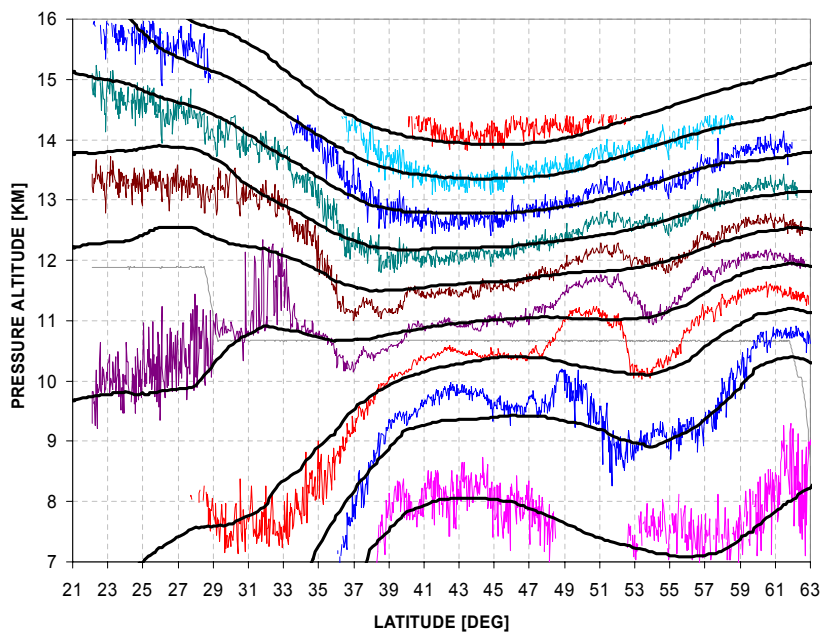

Fig. 2. Overlay of assimilated (thick black traces) and MTPmeasured (thin color traces) IACs for DC-8 flight from Alaska to Hawaii on 11 December 1995. The isentropes are $10 \mathrm{~K}$ apart starting at $310 \mathrm{~K}$ at the altitude/latitude location $8 \mathrm{~km}$ and 43 North. The aircraft altitude (light-gray trace) is $10.7 \mathrm{~km}$ for the flight segment north of 29 degrees latitude, and $11.9 \mathrm{~km}$ for the southern flight segment.

\section{Mid-latitude example of mesoscale versus synoptic isentropes}

The previous example of isentrope "behavior" was at tropical latitudes, using data from the 2-channel MTP. The next example covers a latitude region from Hawaii to Alaska, using measurements of the MTP/DC8. This MTP employs an improved 3-channel radiometer mounted on the NASA DC8 aircraft and flown during 1995/96 flights for TOTE/VOTE (Tropical Ozone Transport Experiment/Vortex Ozone Transport Experiment). The measured isentrope altitude crosssection in Fig. 2 shows much more structure than the assimilated version. In particular, a sub-polar jet has distorted the isentrope field at about 53 degrees north latitude, at $10.5 \mathrm{~km}$. This feature is not present in the synoptic isentropes. The sub-tropical jet produced steeper isentrope surfaces at 35 north latitude, at 10 to $12 \mathrm{~km}$ altitude.

This figure shows that not only do isentropes exhibit mesoscale structure in the real world, but assimilated isentrope altitudes can be in error by as much as $900 \mathrm{~m}$ (e.g., 51 degrees north latitude, 10 to $11 \mathrm{~km}$, and also at 23 degrees latitude, 12 to $16 \mathrm{~km}$ ). Isentropes in the vicinity of the subtropical jet, located at 35 degrees north and $10.4 \mathrm{~km}$ in Fig. 2, are dramatically different when based on the MTP and assimilated synoptic data. Most of the temperature field depicted in this figure is over the ocean, where few radiosonde sites exist. This means that the assimilated temperature field relies more upon satellite soundings than radiosondes, so it is possible that isentropes derived from synoptic temperature fields over land will capture more structure near the short

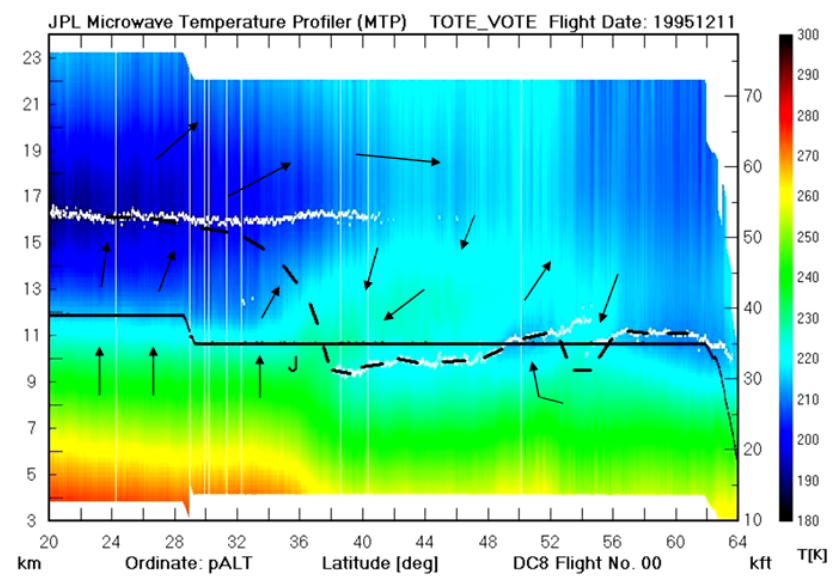

Fig. 3. Color-coded air temperature derived from the MTP/DC8 during a 11 December 1995 flight from Alaska to Hawaii. The arrows are a subjective interpretation of a residual circulation that would produce the measured temperature pattern. Arrow length suggests the magnitude of motion, which in most cases is proportional to the temperature anomaly associated with the arrow. The "J" at 34.5 degrees latitude represents the sub-tropical jet. White dots show MTP-measured tropopause altitudes. The heavy dashed line is a suggested "tracer tropopause" which is based on the MTP tropopause at locations where tracers are compatible with it and is based on either in situ tracers (52-56 degrees) or remotely-sensed ozone (20-36 degrees) elsewhere. The DC- 8 aircraft's altitude is shown by a solid trace $(11.9 \mathrm{~km}$ south of 28 degrees and $10.7 \mathrm{~km}$ north of 28 degrees).

wavelength end of the synoptic representation $(400 \mathrm{~km})$ than is shown here.

Figure 3 is a color-coded display of the MTP temperature field from which the previous IAC was determined. It is presented to lend credibility to the MTP-measured mesoscale features. In the vicinity of the sub-polar jet in situ tracer measurements were used to define the tracer tropopause (ozone mixing ratio of $100 \mathrm{ppbv}$ ), and there is corroboration that the temperature field is distorted at the same location as the tracer field. In the vicinity of the sub-tropical jet both in situ and remote tracers are used to define the tracer tropopause. The Langley Research Center DIAL ozone profiler (Browell, 1989) measured isopleths of ozone mixing ratio, which have been used to define the "tracer tropopause" above the aircraft south of 36 degrees north latitude. By combining in situ and remote tracers it was possible to derive a very steep slope for the tracer tropopause at 38 degrees latitude, just poleward of the jet.

All tracer tropopause behaviors found in this figure are consistent with the expected circulation of air in the vicinity of jets. The black arrows are a subjective suggestion of a "residual circulation" pattern that could produce the temperature departures from a smoother version. The length of the arrows is meant to represent the strength of the motion, which in most cases is determined by the amount of the 


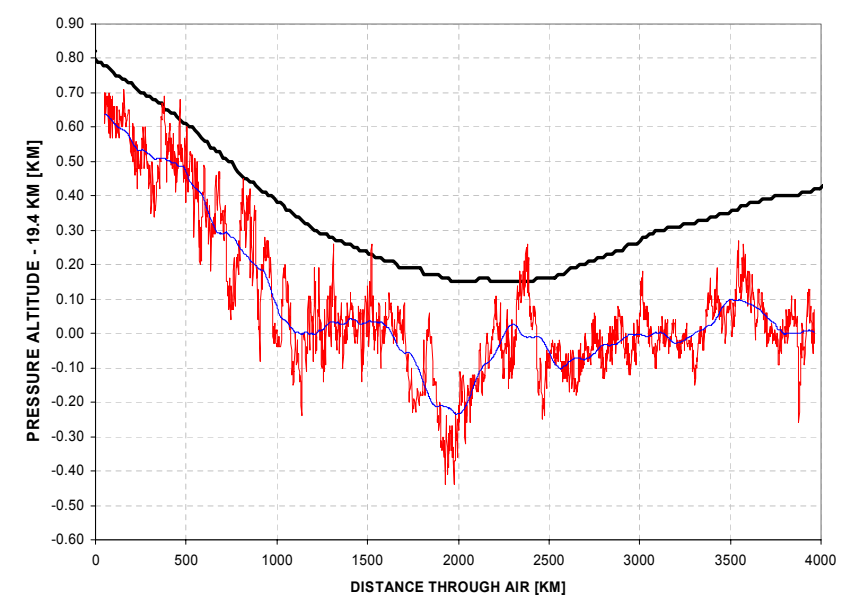

Fig. 4. Two versions of the $490 \mathrm{~K}$ isentrope altitude for the ER-2 flight of 2 November 1991 from California to Maine. The upper trace (black) is based on an assimilated data archive. The thin trace (red) is based on MTP/ER2 measurements. A filtered version of the MTP/ER2 measured trace (blue) is shown to simulate synoptic scale resolution.

temperature anomaly. This figure illustrates the MTP's ability to determine an isentrope field with mesoscale structure that is missing in assimilated temperature fields.

\section{Measured temperature fluctuations for a single isen- trope}

The following cases are based on ER-2 MTP measurements at mid-latitudes. Figure 4 shows a method for studying isentrope structure using the altitude of a single isentrope. The altitude of the $490 \mathrm{~K}$ isentrope is shown for a specific flight (2 November 1991) using two sources: a synoptic scale source (NMC analyzed grid, prepared by Schoeberl et al., 1991) and a mesoscale source (the MTP/ER2). The top trace in Fig. 4 is from a data assimilation and the bottom traces (smoothed and unsmoothed) are from the MTP/ER2. The smoothed version of the MTP/ER2 trace (described in the Appendix) is meant to allow the measurement of two components of error in isentropes based on assimilated data: 1) an offset component, and 2) a missing mesoscale structure component.

For this flight, California to Maine, the ER-2 encountered an eastward wind of $24 \mathrm{~m} / \mathrm{s}$ (ranging from 18 to $30 \mathrm{~m} / \mathrm{s}$ during level flight), which is parallel to the direction of flight. Since an air parcel's vertical excursions can be inferred from isentrope altitude excursions, and assuming the vertical excursions produce adiabatic changes of temperature, the $490 \mathrm{~K}$ isentrope (always within $1 \mathrm{~km}$ of the aircraft) can be used to infer characteristics of air parcel temperature fluctuations. The figure can be used to determine statistical properties of the temperature history of air parcels traveling along the $490 \mathrm{~K}$ isentrope. (It is unimportant for this example that we

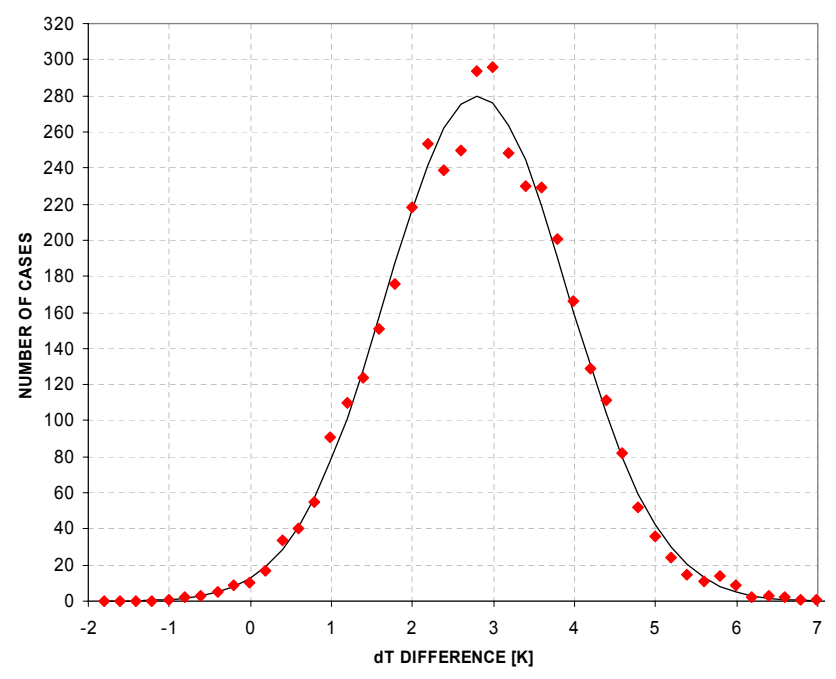

Fig. 5. Histogram of temperature differences corresponding to altitude differences between the measured (mesoscale) and assimilated (synoptic) altitudes in Fig. 4. The model fit is a Gaussian with $\mathrm{FWHM}=2.55 \mathrm{~K}$.

do not know how the isentrope altitude changed shape during its 2-day trip since we are only interested in an assessment of the importance of neglecting mesoscale structures when calculating parcel temperature histories.)

Figure 5 is a histogram of mesoscale deviations from a synoptic scale air parcel temperature history for the 2 November 1991 flight, derived from the differences of the unsmoothed MTP isentrope altitude (lower trace in Fig. 4) from the assimilated data base synoptic scale isentrope altitude (top trace in Fig. 4). The temperature differences histogram can be fitted by an offset term and a Gaussian term. For this case the Gaussian's "full-width at half-maximum" (FWHM) is $2.6 \mathrm{~K}$ and it is offset $2.8 \mathrm{~K}$ from the assimilated temperature history.

Note, however, that some of the $2.6 \mathrm{~K} \mathrm{FWHM}$ is due to "offset wander". The offset wander is actually an error component of synoptic temperature variations based on shortcomings of the assimilated data base. The present study is not concerned with the offset wander error component. Rather, the goal of this study is to evaluate the magnitude of the missing mesoscale temperature fluctuations. Therefore, the desired parameter is the FWHM of mesoscale departures from the smoothed version of the MTP-measured isentrope altitude, converted to temperature units under the adiabatic assumption. This FWHM parameter is $1.6 \mathrm{~K}$. As expected, this is smaller than the $2.6 \mathrm{~K}$ from Fig. 5. For convenience the present analysis has defined "mesoscale fluctuation amplitude", MFA, to be the FWHM of the mesoscale-only temperature fluctuation histogram, where mesoscale departures from synoptic scale are defined using a smoothed-version of the actual (measured) isentrope altitude instead of an isentrope based on the assimilation temperature field. For the example just described MFA=1.6 K. 
Since the adiabatic assumption involves the simple conversion " $1 \mathrm{~km}$ corresponds to $10 \mathrm{~K}$ " MFA can be expressed using either temperature or altitude units. (Note that MFA uses the word "amplitude" to describe a full-width, not a half-width.) Alternative procedures for calculating MFA are given in Appendix A.

\section{Model for mesoscale fluctuation amplitude}

Fractal analysis could be used to assess the amplitude of mesoscale temperature fluctuations, as pointed out by a reviewer of this article (e.g., Tuck et al., 2004; Lovejoy et al., 2004; Mandelbrot, 1998). A fractal analysis solution for a flight segment would yield as a bonus the Hurst exponent, which contains information about the relative importance of long versus short spatial frequencies. For several long flight segments I performed power density spectral analyses and found that in every instance the spectral index was about $-5 / 3$. This also means that the fractal solution "prefactor" should correlate with my chosen independent variables in the same manner as the MFA parameter that I have chosen to use. The additional information provided by the Hurst exponent is unlikely to be useful for the purposes of this investigation, so the simpler analysis upon which the remainder of this section is based should be adequate for present purposes.

In the previous section it was shown that air parcel temperature history for a back trajectory will differ from that calculated from an assimilated field of temperature and wind in ways that can be described as having an offset component (that wanders) and a missing mesoscale component. This investigation is limited to a study of the "missing mesoscale" component. Therefore, the following is an investigation of only the mesoscale fluctuation component, and does not include the "assimilated trajectory altitude wander component." It should be kept in mind that by defining MFA as the FWHM of the distribution of altitude departures of an isentrope surface from a smoothed version of the actual isentrope altitude, MFA will always underestimate mesoscale departures from the back trajectory inferred from an assimilated data base. Mesoscale fluctuations would be as small as MFA only if the assimilated field was perfectly accurate.

This study employs a synoptic averaging procedure that gives approximately the same result as low-pass spatial frequency filtering. An isentrope altitude is averaged using a double boxcar $400-\mathrm{km}$ uniform weighting function, as described in the appendix. Histograms of departures from this synoptic scale representation are then fitted to a Gaussian shape with manual adjustments of offset, height and width, using a spreadsheet application (the use of hand-fitting introduces errors small compared with the property being measured). The resulting MFA determinations are entered into a data base, along with several independent variables: latitude, date, topography type and average altitude of the isentrope. Multiple regression analyses are performed using vari-

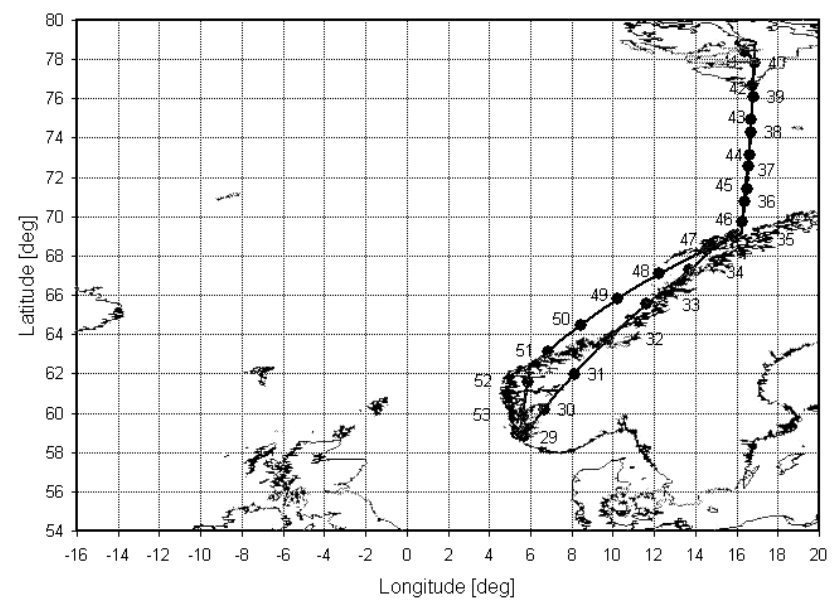

Fig. 6. Flight track for ER-2 flight of 20 January 1989 with markers at $1000 \mathrm{~s}$ intervals of UT.

ous combinations of independent variables, with MFA as the dependent variable.

\subsection{Sample flight}

The following five figures are used to illustrate a typical analysis of a flight and the determination of MFA entries into a data base. Figure 6 is the flight track of a typical ER-2 flight (20 January 1989) used in the present analysis. It occurs under "polar winter" conditions, and is based in Stavanger, Norway. The following list was used to guide the choice for an underlying topography roughness parameter, referred to below as "topography":

$\begin{array}{ll}\text { Land type } & \text { Topography parameter } \\ \text { Ocean } & 0.0 \\ \text { Flat land } & 0.4 \\ \text { Coast } & 0.6 \\ \text { Coastal mountains } & 0.5-0.8 \\ \text { Continental mountains } & 0.6-1.0\end{array}$

The ER-2 flight on 20 January 1989 consists of two types of flight segments, "coastal mountains" and "ocean", with topography scores of 0.8 and 0.0 , respectively. Data are assigned to "coastal mountains" when the ER-2 was within $100 \mathrm{~km}$ of the coast.

Figure 7 is an IAC for this flight, and it is used to choose a specific isentrope to represent flight segments. In this example the $440 \mathrm{~K}$ and $460 \mathrm{~K}$ isentropes are used to represent the first and second halves of the flight (outbound and inbound). The $440 \mathrm{~K}$ isentrope altitude for the first half flight segment is shown in Fig. 8. The thick black trace in this figure is a synoptic scale fit to the MTP data, derived using the double 400$\mathrm{km}$ boxcar procedure. The departures of the thin trace from the thick trace are used to create a histogram of "mesoscale only" fluctuations. Since these data include flight over both "coastal mountains" and "ocean" categories it was necessary 


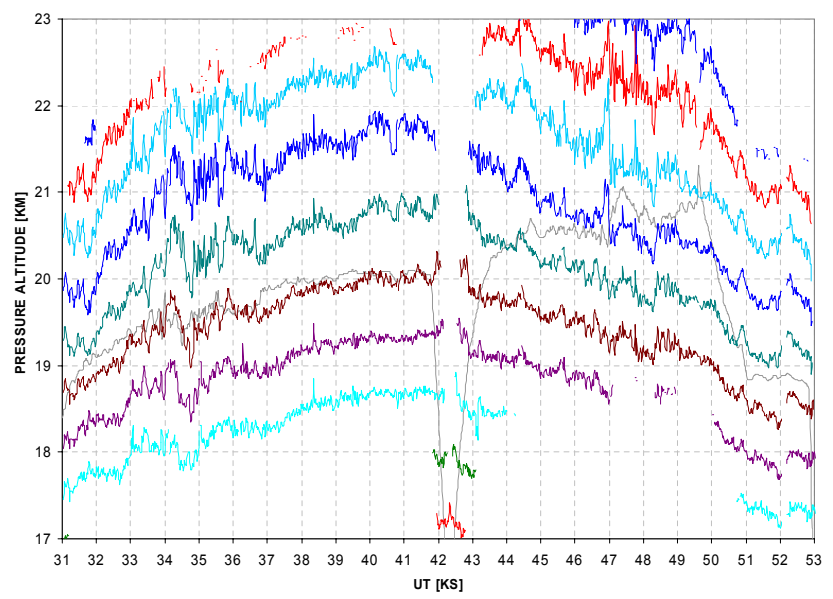

Fig. 7. IAC for ER-2 flight of 20 January 1989 from Stavanger, Norway to Spitzbergen and back. The lowest isentrope is for theta $400 \mathrm{~K}$; the isentrope spacing is $10 \mathrm{~K}$.

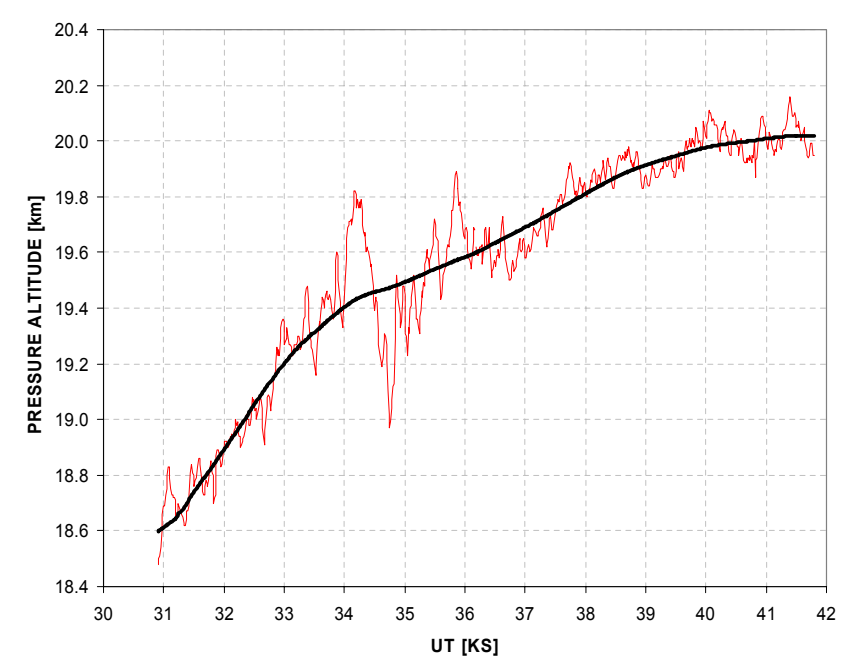

Fig. 8. Altitude of the $440 \mathrm{~K}$ isentrope for the outbound leg of the ER-2 20 January 1989 flight.

to create separate histograms from carefully assigned segments. Examples of the two histograms are shown in Figs. 9a and $b$.

\subsection{ER-2 data}

There are 49 ER-2 flights included in this analysis, from 31 December 1988 to 26 September 2001, yielding 73 MFA values corresponding to a range of latitudes, seasons and underlying topographies. All isentrope altitudes are within the 17 to $21 \mathrm{~km}$ altitude range, with an average altitude of $19.4 \mathrm{~km}$. Figure 10 shows 72 of the 73 MFA values plotted versus latitude (a $360 \mathrm{~m}$ "outlier" associated with a jet stream is not used). The "winter" and "summer" data are plotted with different symbols in this figure, and it is apparent that they vary

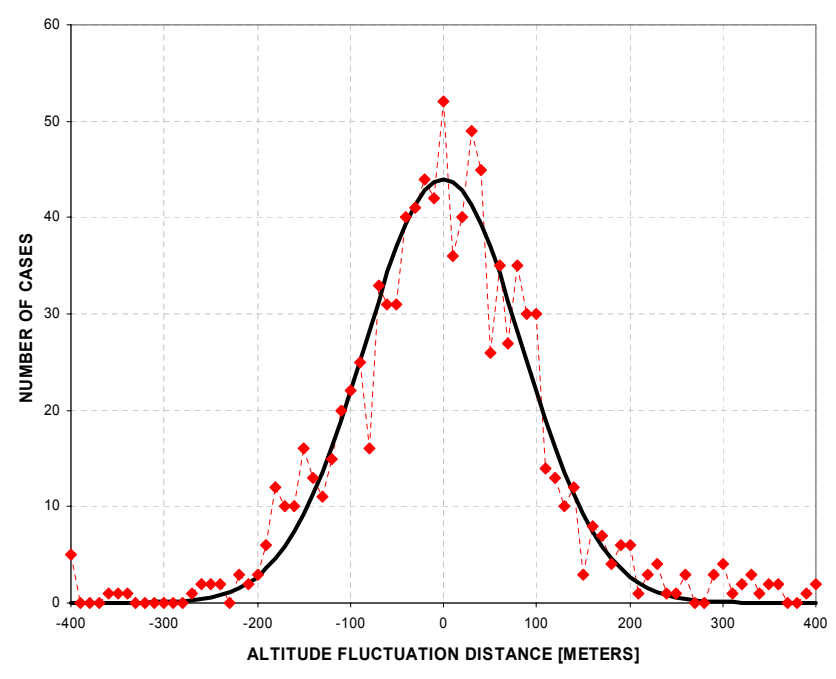

Fig. 9a. Histogram of mesoscale departures from synoptic scale smoothed version of "coastal mountain" portions of the ER-2 20 January 1989 flight.

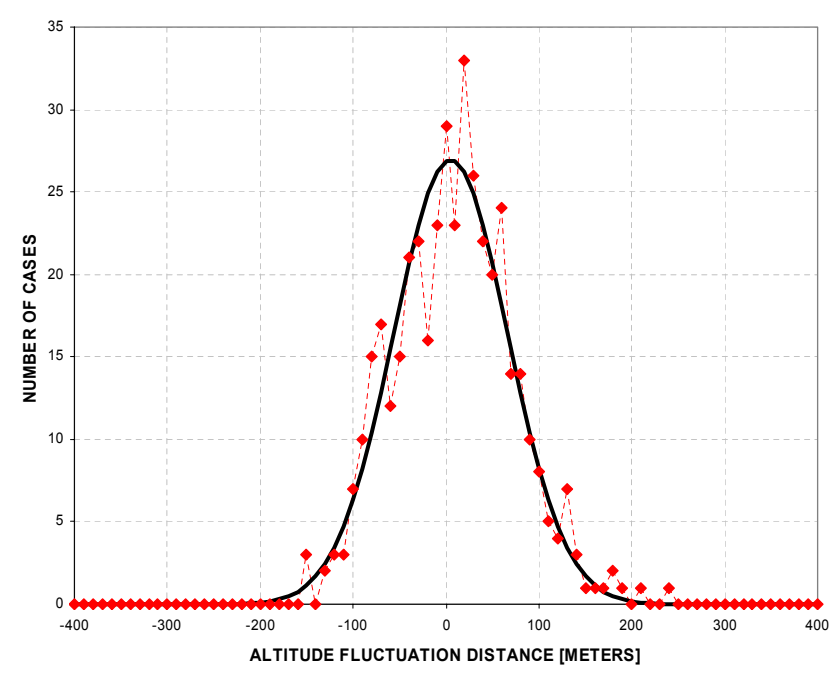

Fig. 9b. Histogram of mesoscale departures from synoptic scale smoothed version of "ocean" portions of the ER-2 20 January 1989 flight.

with latitude differently. MFA is largest at high latitude winter and lowest at high latitude summer. The straight lines correspond to the following equations:

MFA $=120-0.7 \cdot$ Latitude, for summer data

MFA $=120+1.0 \cdot$ Latitude, for winter data

MFA has units of meters and Latitude is in degrees. Whereas there appears to be negligible seasonal variation in the tropics, the amplitude of seasonal variation appears to increase linearly with latitude. In order to sort the data according to season it was necessary to invent a "season parameter" based 


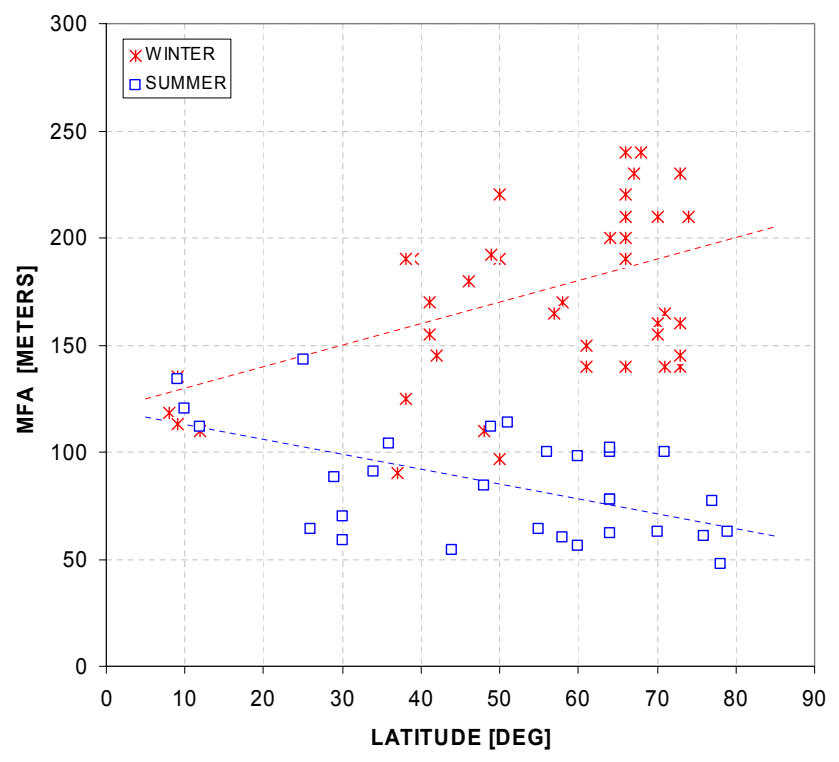

Fig. 10. Measured "Winter" and "Summer" MFA versus latitude. All data are from ER-2 flights.

on the flight date's day of year. The parameter $W$ ("Wintriness") was defined according to the following equation:

$W=1 / 2 \cdot\left[1+\sin \left(\frac{\pi}{2} \cdot \frac{\mathrm{DOY}-295}{365}\right)\right]$

where DOY $=$ day of year. $W$ varies smoothly in a sinusoidal manner from 0.0 on 22 July (one month after summer solstice) to a value of 1.0 on 22 January (one month after winter solstice). In Fig. 10 MFA data are categorized as "summer" or "winter" on the basis of $W$ being less than or greater than 0.5 .

Figure 10 demonstrates that MFA at ER-2 altitudes depends upon latitude and season. A possible dependence upon underlying topography was investigated by performing a 3term least squares (LS) fit of measured MFA versus the following three independent variables: 1) topography parameter, 2) Latitude, and 3) W . Latitude. The following solution was obtained:

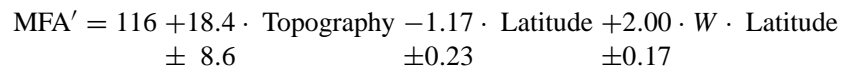

where MFA' [meters] is a model predicted MFA, “Topography" is the underlying topography parameter (ranging from 0 to 1 ), "Latitude" is latitude [degrees] and $W$ is [dimensionless]. Formal standard errors for the coefficient solutions are shown below each coefficient. This fit exhibits $\mathrm{r}^{2}=0.68$, and has a residual MFA of $30.5 \mathrm{~m}$. The "open diamond symbols" in Fig. 11 show the relation between measured MFA and MFA predicted by the above equation.

The four constants in the above Eq. (4) have values significantly different from zero; the ratios for "parameter

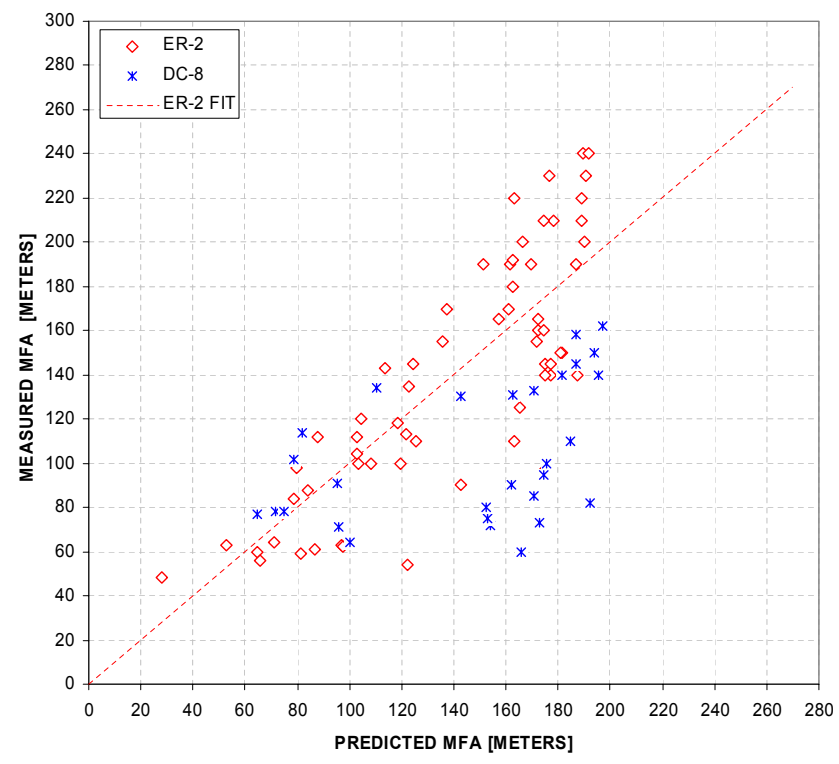

Fig. 11. Measured MFA for ER-2 (open red diamonds) and DC8 (blue X symbols) plotted versus MFA predictions that are based only on ER-2 data with no provision for altitude dependence.

value to parameter value uncertainty" are $2.17,5.08$ and 11.67. (When small adjustments are made for an altitude dependence, described below, this 3-parameter solution is "stronger", yielding a topography "parameter value to uncertainty ratio" of 2.45) All independent variables are statistically significant. From this analysis of ER-2 data it can be concluded that:

1. there is a strong latitude dependence,

2. there is a strong seasonal dependence, and

3. there is a moderately significant topography dependence.

\subsection{DC-8 data and the altitude dependence of MFA}

This section shows that MFA depends upon altitude as well as latitude, season and underlying topography. Since the foregoing analysis was with ER-2 cruise flight data, and is confined to a rather narrow altitude region $(17$ to $21 \mathrm{~km})$, it should be possible to investigate the altitude dependence of MFA by comparing the ER-2 MFA values with MFA results from the lower-flying DC-8 aircraft. Gravity wave theory predicts that mountain wave amplitude should increase with altitude in accordance with the relation:

$A_{i}=A_{0} \cdot\left(D_{i} / D_{0}\right)^{-1 / 2}$

where $\mathrm{A}_{0}$ is an amplitude constant, and $\mathrm{D}_{0}$ and $\mathrm{D}_{i}$ are air density at a standard level and a level of interest. Since air temperature is approximately constant throughout the altitude region between typical DC-8 and ER-2 flight altitudes, 


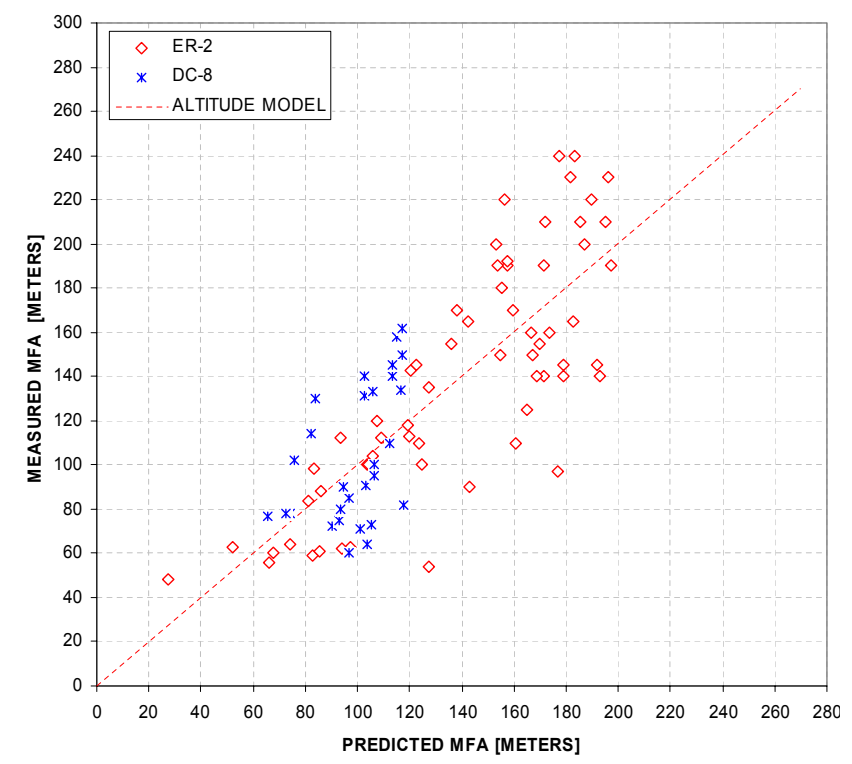

Fig. 12. Measured MFA for ER-2 (red open diamonds) and DC-8 (blue X symbols) plotted versus MFA predictions based on a model that employs an altitude dependence of MFA specified by a pressure exponent of 0.40 .

air density will be approximately proportional to air pressure. This predicted dependence of wave amplitude versus altitude was observed by the ER-2 during encounters with mountain waves over Antarctica (Gary, 1989). What is true of mountain waves may not be true of the ever-present background of gravity waves, so it is necessary to verify the expected dependence of MFA on altitude. To the extent that air density is proportional to air pressure, which it will be when air temperature is uniform between the DC- 8 and ER-2 altitudes, air pressure at flight altitude can be used to represent air density. The following analysis uses air pressure as an independent variable.

Two "outliers" in the DC- 8 MFA data base were removed from consideration due to their association with large amplitude mountain waves and a sub-tropical jet stream. These two DC-8 outliers, and the ER-2 MFA outlier described above, suggest that the MFA models developed in this analysis cannot be used in situations characterized by jet streams or mountain waves.

To illustrate the necessity of invoking an altitude dependence of MFA, the combined ER-2 and DC-8 data sets are plotted against a model that does NOT include an altitude correction term, shown as Fig. 11. The model predictions are clearly too high for the DC-8 MFA data. When a "reciprocal of square-root of pressure" correction is incorporated in the model, in accordance with mountain wave theory, an acceptable plot of measured versus model predicted MFA is produced. This is shown as Fig. 12. A best fit value for the pressure exponent is approximately -0.40 , with an uncertainty that is estimated to be 0.10 (a formal uncertainty could not be obtained for this parameter using the fitting procedure of this analysis). The MFA measurements are compatible with an altitude exponent of -0.50 .

The final equation for representing the combined ER-2 and DC-8 sets of measured MFA involves four independent variables: 1) latitude, 2) a season/latitude parameter, 3) topography, and 4) an "altitude exponent" for use with the ratio of air pressure to a reference air pressure (58.85 millibars, the ER-2 average). The fitting procedure consisted of adopting various values for the altitude exponent and then performing a standard LS fit for the remaining independent variables. The following equation was obtained for relating MFA to the four independent variables:

$$
\begin{array}{cc}
\text { MFA }=(112-1.21 \text { Latitude } & +2.20 \mathrm{~W} \cdot \text { Latitude } \\
\pm 0.27 & \pm 0.20 \\
& \\
+29.0 \text { Topography })(\mathrm{P}[\mathrm{hPa}] / 58.85) & -0.40 \\
\pm 10.2 & \pm 0.10
\end{array}
$$

where MFA has units of meters, $\mathrm{P}$ is air pressure [hPa], and the other parameters are described above. This equation provides a fit to the combined ER-2 and DC- 8 data that exhibits $r^{2}=0.645$ and a residual MFA of $36.6 \mathrm{~m}$.

In every case the uncertainty of the solution coefficient is significantly smaller than the coefficient value, with "value to SE uncertainty" ratios $=4.55,11.27,2.84$ and $\sim 4.3$. The fact that the next-to-last ratio increased from its "ER-2 only" counterpart (i.e., increased from 2.45 to 2.84) shows that the correlation of measured MFA with underlying topography was strengthened by including the DC- 8 data. More data is needed to ascertain the statistical significance of the difference between the altitude exponent fitted solution value of $-0.40 \pm 0.10$ (estimated SE) and the predicted value of -0.50 .

\subsection{Specific procedure for simulating MFA}

At this time Eq. (6) is the best model representing MFA values throughout a range of altitudes, seasons, latitudes and topographies. Using this equation is probably a better alternative than a total disregard of the MFA effect for investigations in which temperature fluctuations are potentially important. This equation also provides a means for superimposing realistic wave amplitudes on a synoptic field intended for use in calculating gravity wave breakdown at high stratospheric altitudes. It has the virtue of not requiring a detailed model for generating the waves and is therefore suitable for operational use.

There are several ways to calculate a specific sequence for "vertical displacement versus horizontal distance", $\mathrm{dZ}(\mathrm{x})$, for adding to a back trajectory calculation of an isentrope surface's altitude. The difficult way is to request a copy (from 
the author) of a program that does this. The program employs an empirical algorithm developed for this purpose. Another way is to request a copy of a file " $\mathrm{dZ}(\mathrm{x})$ " from the author. The user may then modify the $\mathrm{dZ}$ column by a multiplication factor to convert it from having the standard MFA value of $100 \mathrm{~m}$ to the desired MFA. A third way to superimpose mesoscale temperature fluctuations upon a synoptic scale back trajectory is to employ a fractal model using a chosen prefactor and Hurst exponent of $\sim 0.6$, as was done by Murphy (2003). If a specific $\mathrm{dZ}(\mathrm{x})$ function is not required, but a probability density distribution for $\mathrm{dZ}$ is adequate, then this can be easily calculated from the following equation:

$P(d Z)=e^{-(d Z /(0.6 \cdot M F A))^{2}}$

where $\mathrm{dZ}$ is altitude departure from a synoptic average. This equation is normalized such that $\mathrm{P}(0)=1$. MFA must be calculated from Eq. (6) before using this equation.

The MFA values in Table 1 are based on the preceding analysis, and may be convenient for casual users wishing to estimate the possible importance of the MFA effect. To use the table choose a latitude region (left-most column), then choose a season (center two columns), choose an underlying terrain (right-most column), and read an MFA value from the body of the table. This is a "most likely" MFA for ER-2 altitudes $(19.4 \mathrm{~km})$. For DC-8 altitudes, for example, multiply the MFA value by 0.60 . For other altitudes, multiply the MFA value by $(\mathrm{P}[\mathrm{hPa}] / 58.85[\mathrm{hPa}])^{-0.40}$.

\section{Possible cause for observed correlations}

The fact that MFA is greater for "winter high latitudes" suggests that the independent variable created for the multiple regression analysis is merely a "proxy parameter" for wind speed. Support for this comes from the fact that two of the high-value MFA outliers were for flight along jet streams. The fact that clear air turbulence (CAT) occurs preferentially during the winter season, which is conventionally attributed to the greater wind speeds in winter, is also supportive of this interpretation - since CAT occurs when Kelvin-Helmholtz waves are amplified by strong vertical wind shears in the presence of insufficient static stability (provided by the temperature field).

The dependence of MFA on rough underlying terrain seems to require that the source for MFA is air moving over underlying terrain, causing vertical displacements that grow with altitude. This is supported by the near-absence of a correlation of MFA with underlying terrain during the polar summer, when winds are light.

\section{Conclusion}

Back trajectory investigators wishing to assess the magnitude of mesoscale temperature fluctuations may use Eq. (6)
Table 1. MFA for ER-2 Altitudes ( $19.4 \mathrm{~km})$; multiply by 0.58 for DC-8 Altitudes (11.4 km).

\begin{tabular}{llll}
\hline (Latitude region) & Winter & Summer & $\begin{array}{l}\text { (Underlying } \\
\text { terrain) }\end{array}$ \\
\hline POLAR & $239 \mathrm{~m}$ & $68 \mathrm{~m}$ & Mountains \\
& $189 \mathrm{~m}$ & $16 \mathrm{~m}$ & Ocean \\
MID-LATITUDE & $173 \mathrm{~m}$ & $125 \mathrm{~m}$ & Mountains \\
& $121 \mathrm{~m}$ & $72 \mathrm{~m}$ & Ocean \\
TROPICAL & $176 \mathrm{~m}$ & $173 \mathrm{~m}$ & Mountains \\
& $124 \mathrm{~m}$ & $120 \mathrm{~m}$ & Ocean \\
\hline
\end{tabular}

or Table 1 to calculate MFA. This MFA value may be used with Eq. (7) to calculate a probability density function for mesoscale departures to a synoptic record. As an alternative, this MFA value may be used to calculate a fractal sequence (Murphy, 2003). Finally, this MFA value can be used to scale sample sequences of $\mathrm{dZ}(\mathrm{x})$ (available from the author) which can then be added to a synoptic scale version of an air parcel's altitude versus back trajectory distance. Modelers wishing to calculate the altitude where wave breakdown occurs may use this same MFA as a most likely value when it is not feasible to employ an explicit calculation of wave amplitude versus altitude.

The fact that MFA depends upon season, latitude, underlying topography and altitude should provide useful clues for guiding theoretical investigations into the origin of the atmospheric waves producing these ever-present mesoscale fluctuations.

\section{Appendix A}

There are several practical ways to derive MFA besides the one described in the text. They all begin by establishing a smooth line through a measured isentrope surface's altitude versus distance. This can be done by performing a spatial Fourier analysis of the altitude versus distance data (with proper "windowing") and reconstituting the altitude trace after omitting all spatial components with wavelengths shorter than about $400 \mathrm{~km}$. It can also be done by employing a uniform weighting function that is $400 \mathrm{~km}$ wide and sliding it through short flight path distance increments, and then repeating the process. Use of the second boxcar average removes unwanted long wavelength mesoscale spatial frequencies.

Deviations of the unsmoothed data from the smoothed data are used to construct a histogram of mesoscale deviations. A "probable error (PE) difference" can be estimated such that $50 \%$ of actual data exceed this probable difference. This PE value should be multiplied by 3.33 to arrive at the "full-width half-maximum" estimate (which assumes a Gaussian distribution). 
An MFA estimate can also be obtained by noting that $76 \%$ of all data will be contained within a region given by the "average minus MFA" to "average plus MFA."

Or, for one more alternative method, MFA can be computed by multiplying an RMS difference by 2.27. RMS can either be calculated or estimated from the fact that in a normal distribution $68 \%$ of deviations will have an absolute value less than the RMS; the factor 2.27 has been empirically determined for MTP 'data, and is close to the value 2.36 that corresponds to a perfect Gaussian distribution.

To convert an MFA in altitude units to temperature units, divide by $100 \mathrm{~m} / \mathrm{K}$. This conversion is based on the adiabatic assumption that an air parcel's changes in altitude produce temperature changes at the rate of $1 \mathrm{~K}$ per $100 \mathrm{~m}$ (in dry air).

Acknowledgements. The research described in this publication was carried out by the Jet Propulsion Laboratory, California Institute of Technology, under a contract with the National Aeronautics and Space Administration. Specific acknowledgement is made for the contributions of R. Denning for his instrument construction and support throughout all field uses of the Microwave Temperature Profiler. I thank M. J. Mahoney for assuming all MTP responsibilities after my retirement and for his encouragement to bring this work to publication. Appreciation is hereby acknowledged for the use of ozone data produced by E. Browell's DIAL team and for in situ air temperature measurements by R. K. Chan's Meteorology Measurement System group. Finally, acknowledgement is owed the many reviewers who helped identify areas needing improvement.

Edited by: B. Kärcher

\section{References}

Bacmeister, J. T. and Gary, B. L.: Small-Scale Waves Encountered During AASE, Geophys. Res. Lett., 17, 349-352, 1990.

Bacmeister, J. T.: Mountain-Wave Drag in the Stratosphere and Mesosphere Inferred from Observed Winds and a Simple Mountain Wave Parameterization Scheme, J. Atmos. Sci., 50, 377399, 1993.

Browell, E. V.: Differential Absorption Lidar Sensing of Ozone, Proc. IEEE, 77, 419-432, 1989.

Carlsaw, K. S., Wirth, M., Tsias, A., Luo, B. P., Doernbrack, A., Leutbecher, M., Volkert, H., Renger, W., Bacmeister, J. T., Reimer, E., and Peter, T.: Increased stratospheric ozone depletion due to mountain-induced atmospheric waves, Nature, 391(6668), 675-678, doi:10.1038/35589, 1998.

Denning, R. F., Guidero, S. L., Parks, G. S., and Gary, B. L.: Instrument Description of the Airborne Microwave Temperature Profiler, J. Geophys. Res., 94, 16 757-16765, 1989.

Doernbrack, A., Birner, T., Fix, A., Flentje, H., Meister, A., Schmid, H., Browell, E., and Mahoney, M. J.: Evidence for Inertia-Gravity Waves Forming Polar Stratospheric Clouds Over Scandinavia, J. Geophys. Res., 107(D20), 8287, doi:10.1029/2001JD000452, 2002.

Farman, J. C., Gardner, B. G., and Shanklin, J. D.: Large Losses of Total Ozone in Antarctica Reveal Seasonal ClOx/Nox Interaction, Nature, 315, 207-210, 1985.
Fueglistaler, S., Buss, S., Luo, B. P., Wernli, H., Flentje, H., Hostettler, C. A., Poole, L. R., Carslaw, K. S., and Peter, T.: Detailed Modeling of Mountain Wave PSCs, Atmos. Chem. Phys., 3, 697712, 2003, http://www.atmos-chem-phys.net/3/697/2003/.

Gage, K. S. and Nastrom, G. D.: Spectrum of Atmospheric Vertical Displacements and Spectrum of Conservative Scalar Passive Additives Due to Quasi-Horizontal Atmospheric Motions, J. Geophys. Res., 92, 13 211-13216, 1986.

Gao, R. S., Richard, E. C., Popp, P. J., et al.: Observational Evidence for the Role of Denitrification in Arctic Stratospheric Ozone Loss, Geophys. Rev. Lett., 28, 2879-2882, 2001.

Gary, B. L.: Observational Results Using the Microwave Temperature Profiler During the Airborne Antarctic Ozone Experiment, J. Geophys. Res., 94, 11 223-11 231, 1989.

Hoyle, C. R., Luo, B. P., and Peter, T.: The Origin of High Ice Crystal Number Densities in Cirrus Clouds, J. Atmos. Sci., 62, 2568-2579, 2005.

Karcher, B. and Strom, J.: The roles of dynamical variability and aerosols in cirrus cloud formation, Atmos. Chem. Phys., 3, 823838, 2003, http://www.atmos-chem-phys.net/3/823/2003/.

Lovejoy, S., Schertzer, D., and Tuck, A.: Fractal aircraft trajectories and nonclassical turbulent exponents, Phys. Rev. E, 70, 036306 , 2004.

Mandelbrot, B. B.: Fractals and 1/f Noise, Berlin, Springer, 1998.

Murphy, D. M.: Dehydration in cold clouds is enhanced by a transition from cubic to hexagonal ice, Geophys. Res. Lett., 30, 2230, doi:10.1029/2003GL018566, 2003.

Mahoney, M. J. and Gary, B.: DC-8 MTP Calibration for SOLVE-2, SOLVE-2/Vintersol Science Team Meeting, Orlando, FL, 21-24 October 2003.

Murphy, D. M. and Gary, B. L.: Mesoscale Temperature Fluctuations and Polar Stratospheric Clouds, J. Atmos. Sci., 52, 17531760, 1995.

Nastrom, G. D. and Gage, K. S.: A Climatology of Atmospheric Wave Number Spectra Observed by Commercial Aircraft, J. Atmos. Sci., 42, 950-960, 1985.

Schoeberl, M., Newman, P., and Lait, L.: Goddard Space Flight Center Code 916, XS mission exchange files, 1991 and 1994.

Scott, S. G., Bui, T. P., Chan, K. R., and Bowen, S. W.: The Meteorological Measurement System on the NASA ER-2 Aircraft, J. Atmos. Oceanic Technol., 7, 525-540, 1990.

Tabazadeh, A., Toon, O. B., Gary, B. L., Bacmeister, J. T., and Schoeberl, M. R.: Observational Constraints on the Formation of Type Ia Polar Stratospheric Clouds, Geophys. Res. Lett., 23, 2109-2112, 1996.

Tuck, A. F., Hovde, S. J., and Bui, T. P.: Scale invariance in jet streams: ER-2 data around the lower-stratosphere polar night vortex, Quart. J. Meteorol. Soc., 130, 2423-2444, 2004.

Voigt, C., Tsias, A., Doernbrack, A., Meilinger, S., Luo, B., Schreiner, J., Larsen, N., Mauersberger, K., and Peter, T.: Nonequilibrium compositions of liquid polar stratospheric clouds in gravity waves, Geophys. Res. Lett., 27(23), 3873-3876, doi:10/1029/2000GL012168, 2000.

Wofsy, S. C., Gobbi, G. P., Salawich, R., and McElroy, M. B.: Vapor Pressure of Solid Hydrates of Nitric Acid: Implications for Polar Stratospheric Clouds, Science, 259, 71-74, 1993.

Wu, D. L. and Waters, J. W.: Satellite Observations of Atmospheric 
Variances: A Possible Indication of Gravity Waves, Geophys. Res. Lett., 23, 3631-3634, 1996.
Wu, D. L. and Waters, J. W.: Gravity-Wave-Scale Temperature Fluctuations Seen by the UARS MLS, Geophys. Res. Lett., 23, 3289-3292, 1996. 